\title{
618 EXPANDING CANCER IMMUNOTHERAPY BY EXPLOITING RECALL IMMUNITY - A NEW CO-THERAPY OPTION FOR CHECKPOINT INHIBITORS
}

Kathlynn Brown, Michael McGuire, Anuja Pande, Indu Venugopal ${ }^{*}$. SRI International, Rockingham, VA, USA

Background Immune checkpoint inhibitors (CIs) have emerged as a revolutionary treatment for several cancer types. Despite significant improvement in prognosis for some patients, there are associated challenges. CIs do not work well on immunecold tumors, thereby eliciting an insufficient immune response. They are also not as effective in tumors with low mutational burden due to dependance on tumor self-antigens for immune recognition. Therefore, there is a need for a solution to improve the efficacy of CIs to make them applicable to the entire cancer patient population.

Methods To address this challenge, we have developed a novel immunotherapy capable of delivering previously encountered antigenic peptides specifically to cancer cells and facilitating their presentation through the MHC class I pathway. Our therapy utilizes a synthetic nanoparticle delivery system comprising of three components: a neutral stealth liposome, an encapsulated synthetic immunogenic HLA class I restricted peptide derived from measles virus (MV), and a tumor-targeting peptide on the external surface of the liposome. The targeting peptide results in accumulation of the liposomes specifically inside cancer cells, and facilitates presentation of the MV-derived immunogenic peptides in HLA class I molecules. We refer to this system as TALL (Targeted Antigen Loaded Liposomes). As a result, TALL can generate a strong secondary immune response specifically against the targeted tumor cells in a patient who has been previously vaccinated against or infected by MV. In short, we are attempting to trick the immune system into responding as though the cancer cell is infected with MV without the use of a viral particle. Advantageously, as TALL can provide a potent synthetic antigen specifically to tumor cells, it can convert immune-cold tumors into immune-hot, resulting in a robust cytotoxic $\mathrm{T}$ lymphocyte response. Therefore, we conducted pilot studies to determine the efficacy of combining TALL with the anti-PD1 checkpoint inhibitor.

Results Treatment with TALL alone substantially reduces growth of lung, triple-negative breast, and pancreatic tumors in mice. Treatment with TALL and CI combination therapy showed at least a 10-fold reduction in tumor burden in mice bearing orthotopic breast and pancreatic tumors when compared to using CI treatment alone. The combination treatment also successfully prevented metastasis from occurring.

Conclusions TALL can successfully be used in combination with existing immunotherapies like checkpoint inhibitors, to generate a robust cytotoxic $\mathrm{T}$ lymphocyte response directed specifically against the tumor, resulting in a drastic reduction of tumor burden.

http://dx.doi.org/10.1136/jitc-2021-SITC2021.618 